\title{
Spectral Efficient Coding Schemes in Optical Communications
}

\author{
T. H. Lotz ${ }^{*}$ W. Saue r-Gre ff, R. Urbansky
}

University of Kaiserslautern, Department of Electrical and Computer Engineering, Kaiserslautern, 67663, Germany

\begin{abstract}
Achieving high spectral efficiency in optical transmissions has recently attracted much attention, aiming to satisfy the ever increasing demand for high data rates in optical fiber communications. Therefore, strong Forward Error Correction (FEC) coding in combination with multilevel modulation schemes is mandatory to approach the channel capacity of the transmission link. In this paper we give design rules on the joint optimization of coding and signal constellations under practical considerations. We give trade-offs between spectral efficiency and hardware comple xity, by comparing coding schemes using capacity achieving constellations with bit-interleaved coded modulation and iterative decoding (BICM-ID) against applying conventional square quadrature amplitude modulation (QAM) constellations but emp loying powerful low complexity low-density parity-check (LDPC) codes. Both schemes are suitable for optical single carrier (SC) and optical orthogonal frequency-division multiplexing (OFDM) transmission systems, where we consider the latter one in this paper, due to well-studied equalization techniques in wireless communications. We numerically study the performance of different coded modulation formats in optical OFDM transmission, showing that for a fiber optical transmission link of $960 \mathrm{~km}$ reach the net spectral efficiency can be increased by $\approx 0.4 \mathrm{bit} / \mathrm{s} / \mathrm{Hz}$ to $8.61 \mathrm{bit} / \mathrm{s} / \mathrm{Hz}$ at a post FEC BER of $<10^{-15}$ by using coded optimized constellations instead of coded 64-QAM.
\end{abstract}

Keywords Coherent Communications, Optical OFDM, Bit-Interleaved Coded Modulation and Iterative BICM-ID, Spectral Efficiency, Capacity Approaching Constellations (IPM)

\section{Introduction}

Over the last decade there has been an exponential growth of bandwidth-intensive services such as video on demand, cloud storage and social networking which require large volumes of data to be transmitted over long distances. Based on current trends, this growth is like wise to continue, driving the need to increase transmission capacity. Ongoing commercial developments with data rates of $100 \mathrm{Gbit} / \mathrm{s}$ in the standardized $50 \mathrm{GHz}$ wavelength-division multiplexing (WDM) grid consider polarization division multiplexed quadrature phase shift keying (PDM-QPSK) as the most suitable modulation format[1]. This allows a per polarization spectral efficiency of up to $2.0 \mathrm{bit} / \mathrm{s} / \mathrm{Hz}$. Next generation systems will need to supply data rates beyond $400 \mathrm{Gbit} / \mathrm{s}$. Therefore it is necessary to exp loit the channel capacity of the fiber up to its theoretical limit. The most effective method to increase the spectral efficiency of any communication system is to employ larger modulation formats[2]. However, in order to approach channel capacity the constellation itself needs to be modified with respect to

* Corresponding author:

lotz@eit.uni-kl.de (T. H. Lotz)

Published online at http://journal.sapub.org/ijoe

Copyright (C) 2012 Scientific \& Academic Publishing. All Rights Reserved information theory. $\operatorname{In}[3]$ the design principles of capacity achieving circular constellations, based on iterative polar quantization (IPQ), are proposed. For higher modulation formats the robustness to noise is degraded, claiming the need for powerful FEC coding schemes, relying on the "Turbo Principle"[4]. The combination of iterative polar modulation (IPM) and LPDC codes have already been a matter of investigation in[5], using multilevel coding (MLC) and iterative multistage decoding[6], but omitting complexity considerations, investigations on the available channel capacity and error floor evaluations. To cope with the non-linear channel effects and dispersion, we consider OFDM in combination with multiple-input multiple output polarization multip le xed equalization to be most appropriate, as it offers high spectral efficiency and needs only simple computation operations for equalization[7].

In this paper we propose a high spectral efficient coded modulation scheme for implementation in future optical communication systems operating at data rates beyond $400 \mathrm{~Gb} / \mathrm{s}$. In detail, we adapt the "Turbo Principle" to BICM-ID $[8]$ and comb ine it with a high-rate outer algebraic code to obtain a post-FEC BER $<10^{-15}$, which is a typical demand in optical transponders. Furthermore we give simple design principles for the design of BICM-ID based on the extrinsic information transfer (EXIT) chart analysis[9]. The optical channel is considered to be 
weakly-nonlinear. Therefore the proposed techniques are also applicable for single-carrier transmission; however we consider OFDM since it appears to be more appropriate for the high order modulation formats and efficient equalization algorithms that are well established in wireless communications.

The remainder of the paper is organized as follows: In Section 2 the design principles of capacity achieving constellations are derived. In Section 3, the investigated optical OFDM system is introduced and the available channel capacity for different modulation formats is estimated. The basic coded modulation scheme, namely BICM-ID, is reviewed in Section 4. Design principles for BICM-ID with IPQ signal constellations, using recursive systematic convolutional (RSC) codes and square signal constellations, using LDPC codes, are given in Sections 5 and 6. We conclude our paper in Section 7.

\section{Design of Capacity Approaching Constellations by Means of Iterative Polar Quantization}

In order to explo it the available channel capacity up to its theoretical limit the signal distribution needs to comply with the channel characteristics. In additive wh ite Gaussian noise (AWGN) channels, such as thermal and amplified spontaneous emission (ASE) noise dominated channels, a Gaussian source provides the maximum shaping gain value.

A Gaussian distribution at the channel input can be realized by different strategies. One considers shaping, first proposed in[10] by Gallager, where it is shown that optimal coding for the Gaussian channel is utilized by grouping bits fro m a binary code, where the larger group is assigned to the less probable points in the constellation, and the smaller to the more frequent[11]. In this concept the basis constellation A is square; shaping is performed by additional shaping encoders and decoders. A hardware complexity considering design of this approach is proposed in[11], but is hardly feasible for high data rates.

Therefore we consider a different strategy to provide a Gaussian source, by applying non-uniform signal constellations, which comply with the channel characteristics. Design principles of capacity achieving constellations are proposed in[3]. In order to provide Gaussian signaling at the input of the system, we optimize the signal constellation A by minimizing the quantization mean square error (QMSE) of a Gaussian information source by means of IPQ. The constellation points of the obtained constellation are distributed over the circles of radii determined by a Rayleigh distribution[3]. The idea of using a Rayleigh distribution $p(r)$ originates from the fact that the envelope $r$ of a two-dimensional Gaussian distribution is Rayleigh distributed. Let $N_{r}$ denote the number of circles in the constellation and $L_{j}$ denote the number of constellation points per circle of radius $m_{j} ; j \in\left\{1,2, \ldots, N_{r}\right\}$. Then the optimu mu mber of constellation points at the $j$ th circle for a
$2^{M}$-ary signal set is determined by minimizing the QMSE



The radius of the $j$ th circle calculates to

$$
m_{j}=\left[2 \sin \left(\Delta \theta_{j} / 2\right) \int_{r_{j}}^{r_{j}+1} p(r) d r\right] /\left[\Delta \theta_{j} \int_{r_{j}}^{r_{j}+1} p(r) \mathrm{d} r\right] .
$$

where $\Delta \theta_{j}=2 \pi / L_{j}$. The limits of integration in (1) and (2) are determined by

$$
r_{j}=\frac{\pi\left(m_{j}^{2}-m_{j-1}^{2}\right) / 2}{m_{j} L_{j} \sin \left(\Delta \theta_{j} / 2\right)-m_{j-1} L_{j-1} \sin \left(\Delta \theta_{j-1} / 2\right)} .
$$

The optimu m signal constellation $\boldsymbol{A}$ for a $2^{M}$-ary signal set is found by applying equations (1)-(3) in an iterative fashion until convergence[3]. Table 1 summarizes the details of the 64-IPM constellation, for other constellation sizes see[5] and[12].

Table 1. Summary of the specifications for the 64-IPM

\begin{tabular}{|c|c|c|c|c|c|c|}
\hline & \multicolumn{6}{|c|}{$2^{\mathrm{M}}=64$} \\
\hline$N_{r}$ & 1 & 2 & 3 & 4 & 5 & 6 \\
\hline$r_{j}$ & 0 & 0.543 & 1.019 & 1.549 & 2.246 & 4.5 \\
\hline$m_{j}$ & 0.334 & 0.78 & 1.263 & 1.835 & 2.614 & \\
\hline$L_{j}$ & 5 & 11 & 15 & 17 & 16 & \\
\hline
\end{tabular}

Figure 1 depicts the 64-IPM constellation as specified in Table 1.

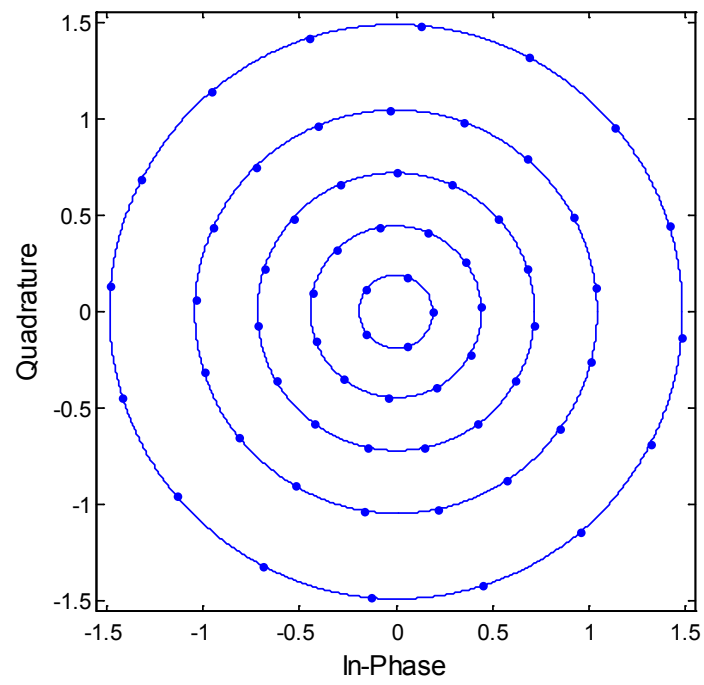

Figure 1. The 64-IPM signal constellation

The distribution of the points is non-uniform and forms a discrete 2-D Gaussian distribution with zero mean. Since the applied mapping is crucial for the performance of BICM-ID, we designed the mapping to be most Gray like, i.e. the binary decomposition of neighboring symbols differs in a minimum number of dig its. A pure Gray labeling is not available for constellations designed by IPQ, due to a non-uniform symbol spacing and differing numbers of neighboring symbols.

The benefit of applying IPM constellations over QAM constellations is revealed in Figure 2, showing the spectral 
efficiency of QAM and IPM, which was calculated by

$$
C(\mathrm{~A})=\int_{\mathrm{C}} \frac{1}{|\mathrm{~A}|} \sum_{\underline{a}_{m} \in \mathrm{A}} f_{r}\left(\underline{r} \mid \underline{a}_{m}\right) \log _{2} \frac{f_{r}\left(\underline{r}^{\prime} \mid \underline{a}_{m}\right)}{\frac{1}{|\mathrm{~A}|} \sum_{\underline{a}_{k} \in \mathrm{A}} f_{r}\left(\underline{r} \mid \underline{a}_{k}\right)} \mathrm{d} r .
$$

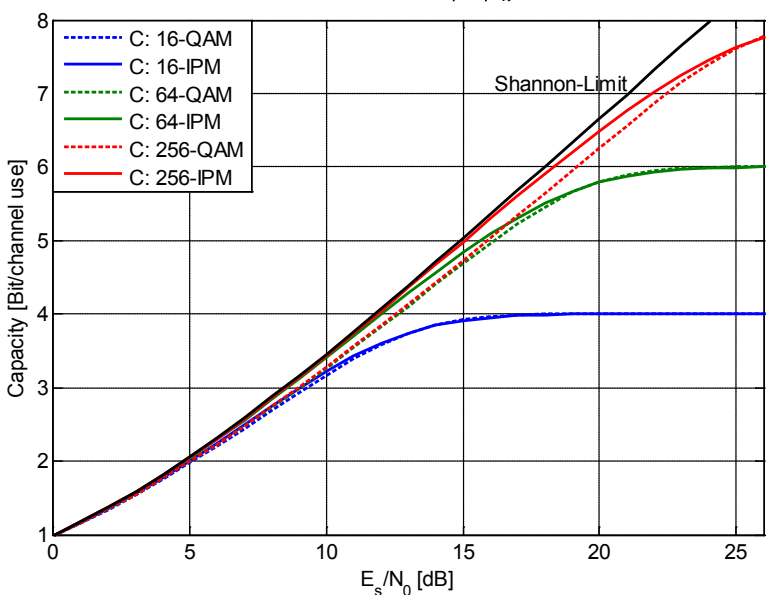

Figure 2. Channel capacities for different IPM and square QAM modulations

We assumed equal a-priori probability for the input symbols $\underline{a}_{m} \in \mathbf{A}, p(\underline{a})=1 /|\mathbf{A}|$ and use the conditional probability density function (PDF) of the complex valued AWGN channel

$$
f_{r}(\underline{r} \mid \underline{a})=\frac{1}{2 \pi \sigma^{2}} \exp \left(-\frac{1}{2 \sigma^{2}}|\underline{r}-\underline{a}|^{2}\right),
$$

where the variance $\sigma^{2}$ corresponds to the double-sided noise power spectral density $N_{0} / 2$. Obviously, IPM signal constellations approach capacity for low and medium signal-to-noise ratios and outperform conventional square-QAM constellations.

\section{The Optical OFDM System and Channel Capacity Estimation}

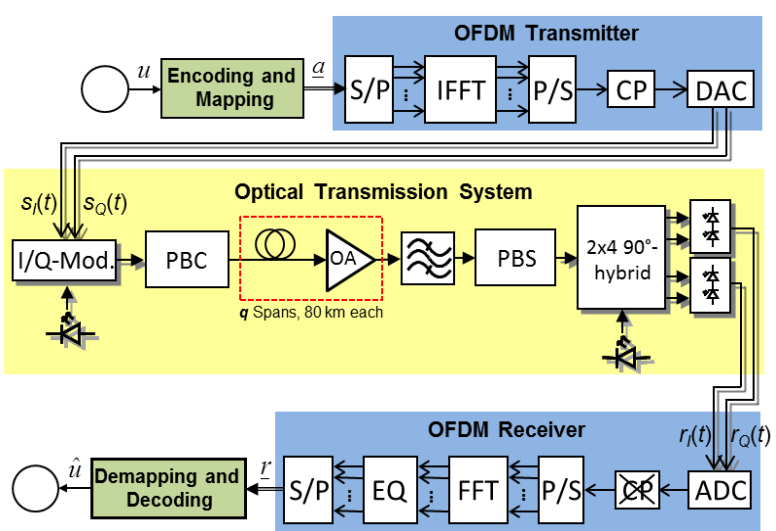

Figure 3. The optical OFDM communication system

The objective of this paper is to provide practical design principles for capacity approaching coding schemes. Therefore knowledge of the available channel capacity is inevitable. The optical OFDM system under investigation is depicted in Figure 3.
A binary information sequence $\boldsymbol{u}$ is encoded and mapped to complex valued $2^{M}$-ary symbols $\underline{a}$. After mapping to a block of symbols, the symbols $\underline{a}$ are fed to an OFDM transmitter, electro-optical I-/Q-modulated, polarization multiplexed and transmitted over the fiber-optical channel. At the receiver, the signal is demultiplexed, coherently demodulated sampled and equalized in the frequency domain, providing the complex samples $\underline{r}$ at the output of the OFDM receiver. For a description of our OFDM system in detail see[13].

In the simulations of the optical system we modulate 864 subcarriers within a bandwidth of $24.1 \mathrm{GHz}$ in each state of polarization. The cyclic prefix length equals to $1 / 10$ of the original OFDM symbol duration (not considered in the capacity estimation). The lasers line-width is assumed to be $100 \mathrm{kHz}$. The transmission link itself consists of $q=12$ identical spans of standard single mode fiber (SSMF) (length: $80 \mathrm{~km}$, chromatic dispersion coefficient: $17 \mathrm{ps} / \mathrm{nm} / \mathrm{km}$, attenuation: $0.2 \mathrm{~dB} / \mathrm{km}$ ); the whole transmission link covers $960 \mathrm{~km}$. The per-channel optical input power was varied from -10 to $0 \mathrm{dBm}$. The simulation model of the optical channel considers the Kerr effect with the non-linear coefficient $1.33 / \mathrm{W} / \mathrm{km}$; polarization dependent loss is neglected. Optical amplifiers (OA) compensate for attenuation; their noise-figure is $4 \mathrm{~dB}$. Further we include five WDM channels at a $25 \mathrm{GHz}$ grid in our simu lations. The receiver contains a $30 \mathrm{GHz}$ optical pass-band filter and a 6 bit analog-to-digital converter (ADC).

Studies about the noise characteris tics show that the whole setup can be treated as weakly non-linear, so the Gaussian PDF of equation (5) is appropriate to describe the channel with respect to the OFDM subcarriers. Using (4) and (5), the information spectral densities of the resulting channel for various per-WDM channel input powers and different modulations format were estimated. The results are shown in Figure 4.

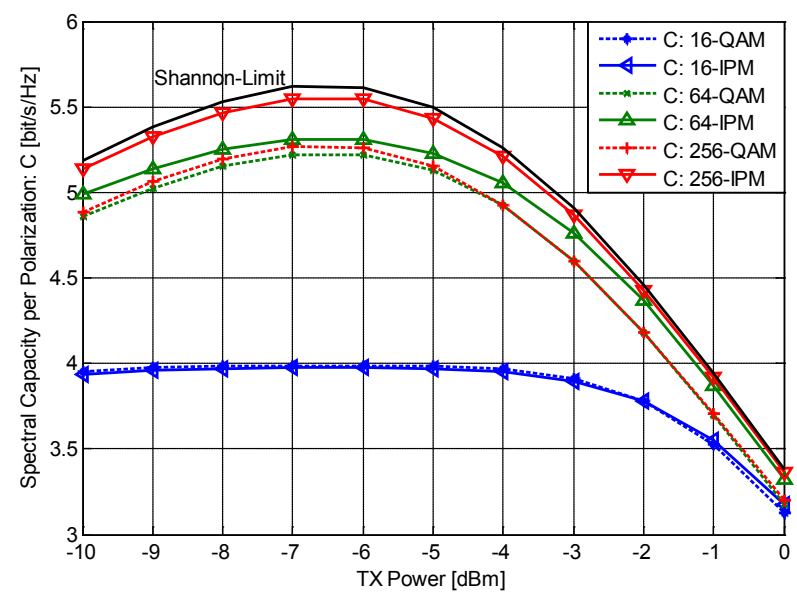

Figure 4. Capacities of different modulation formats after $960 \mathrm{~km} \mathrm{SSMF}$ transmission

Using OFDM, the complex-valued signal transmitted through the optical system can be assumed to be approximately 2-D Gaussian distributed. Therefore, from 
information theory, the signal meets the requirements to approach the channel capacity of the resulting optical channel mainly corrupted by additive Gaussian noise. On the other hand, although we are using an OFDM system, the achievable capacity for IPM on each subcarrier is closer to the Shannon limit than for conventional QAM, for two reasons: First, the average symbol power is decreased and second, regarding the OFDM system as part of the channel, the optimum symbol constellation has to be Gaussian, due to the fact that the symbols $\underline{r}$ at the receiver side after the FFT are corrupted only by fairly Gaussian distributed noise, no matter what distortions occurred on the optical channel, if the number of subcarriers is large. As displayed in Figure 4, the optimum fiber launch power is $-7 \mathrm{dBm}$. For low optical input powers, ASE noise is dominant, the signal-to-noise ratio (SNR) increases by $1 \mathrm{~dB}$ per $1 \mathrm{~dB}$ input power increment (as expected for a linear system with AWGN). But when the optical power is increased above a certain threshold, the Kerr effect becomes dominant, causing an SNR degradation of 2 $\mathrm{dB}$ per $1 \mathrm{~dB}$ power increment[13]. The threshold for the Kerr effect to become dominant is at a fiber launch power of -7 $\mathrm{dBm}$, which corresponds to an $\mathrm{E}_{\mathrm{s}} / \mathrm{N}_{0}$ of $16.9 \mathrm{~dB}$ in an $\mathrm{AW} \mathrm{GN}$ channel model, supplying a channel capacity of 5.61 $\mathrm{bit} / \mathrm{s} / \mathrm{Hz}$.

Obviously only the 256-IPM signal constellation is able to provide a capacity close to the channel capacity, leaving a gap of only $0.07 \mathrm{bit} / \mathrm{s} / \mathrm{Hz}$. Unfortunately for this constellation a hardware implementation currently seems unfeasible and further we do not expect that switching from 64-IPM to 256-IPM is justifiable, since it requires an ADC of more than 6 bit and a high code overhead. Therefore we restrict us in the following on the design of coded modulation for 64-IPM and for comparison 64-QAM, supplying an information spectral density of $5.31 \mathrm{bit} / \mathrm{s} / \mathrm{Hz}$ and $5.22 \mathrm{bit} / \mathrm{s} / \mathrm{Hz}$, respectively, which is still close to the Shannon limit.

\section{Advanced Spectral Efficient Coding in Optical Communications}

In classical non-coherent optical co mmunication systems, operating at data rates up to $10 \mathrm{~Gb} / \mathrm{s}$, no powerful FEC coding is required, since in the systems a pre-FEC BER of $<10^{-4}$ is obtained and a post-FEC BER of only $<10^{-12}$ is demanded. Hence the algebraic Reed-Solomon (RS) (255, 239) code, supplying a net coding gain (NCG) of $5.62 \mathrm{~dB}$ at a pre-FEC BER of $1.8 \cdot 10^{-4}$ for a post-FEC BER $10^{-12}$ is the most used code in these systems [14].

But when higher data rates are claimed, more powerful coding schemes need to be considered. In today's systems with $40 \mathrm{Gbit} / \mathrm{s}$ differential phase-shift keying, enhanced FEC (EFEC) coding, as recommend by the ITU in [15], is utilized by the concatenation of the Bose-ChaudhuriHocguenghem $(\mathrm{BCH})(3860,3824)$ code and the $\mathrm{BCH}$ (2040, 1930) code, using iterative decoding. The $\mathrm{BCH}$ $(3860,3824)+\mathrm{BCH}(2040,1930)$ concatenated code has a code overhead of $6.69 \%$. After three iterations this code achieves a NCG of $8.99 \mathrm{~dB}$ at a pre-FEC BER of $3.15 \cdot 10^{-3}$ and a post-FEC BER of $10^{-15}$. If iterative decoding is to be avoided the ITU recommends the RS (2720, 2550) code with a NCG of $8 \mathrm{~dB}$ at a pre-FEC BER of $1.1 \cdot 10^{-3}[15]$. A 11 of these codes are binary linear cyclic codes, with decoders processing hard decision values of the received output bit stream. So these schemes are most suitable for low BER, but when coherent modulation formats beyond QPSK are employed and approaching the Shannon limit is desired, soft decision decoding is inevitable.

A straightforward approach to combine multilevel signaling and coding is BICM-ID, which can be considered the simplest approach to achieve high spectral efficiency while providing a low decoder complexity[2]. However, coding schemes relying on the Turbo Principle may run into an error floor, which is several decades higher than the desired post-FEC BER of $10^{-15}$. Therefore, we apply BICM-ID for inner coding and concatenate it with a high-rate outer code to remove residual errors. The proposed coding scheme is depicted in Figure 5.

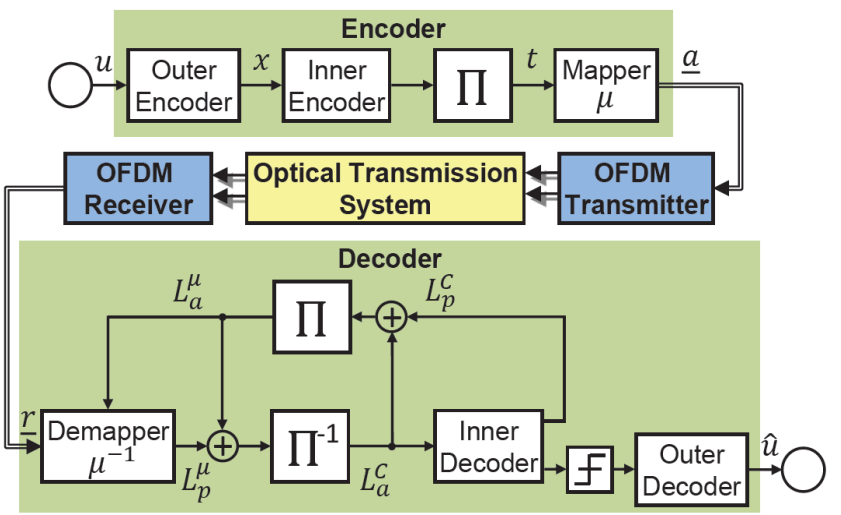

Figure 5. Proposed concatenated it erative soft-demapping and decoding scheme

In the following, we consider the binary RS $(2720,2550)$ code with a code rate of $R_{R S}=0.9375$ and a length of 32640 bit for outer coding. The concept inner coded modulation scheme is as follows: The transmission is performed on a block basis: $k$ bits of the outer encoded sequence $x$ are fed to a recursive systematic convolutional (RSC) or LDPC encoder, which extends the sequence by $n-k$ parity bits, where $n$ is the codeword (CW) length. As explained later, we prefer to use punctured RSC codes for IPM signal constellations and LDPC codes for square QAM signal constellation, see Section 5 and 6.

After encoding the $\mathrm{CW}$ is fed to a row column interleaver, so the bits in the binary decomposition of a symbol are uncorrelated, which is a pre-requirement for iterative demapping and decoding. After interleaving the grouping operation takes $M$ coded bits $t_{0,1, \ldots, M-1} \in\{0,1\}$ to form the complex output symbol $\underline{a}=\operatorname{map}\left(t_{0,1, \ldots, M-1}\right)$. This mapping operation map $(\cdot)$ is essential for the iterative demapping and decoding process, since it links up the bits $t_{0,1, . ., M-1}$ to a symbol and mutual dependencies arise between them[8].

After mapping the complex symbols $\underline{a}$ are transmitted 
over the channel. In our optical OFDM system we use a high number of subcarriers; therefore the central limit theorem applies when performing the FFT on the received channel symbols, causing that after equalization the symbols $\underline{r}$ at the input of the decoder are only corrupted by fairly Gaussian distributed noise; $\underline{r}=\underline{a}+\underline{n}$, where $\underline{n}=n_{I}+$ $j n_{Q}$, with $n_{I}, n_{Q}$ being realizations of two independent Gaussian random variables and variance $\sigma^{2}=\sigma_{I}^{2}=\sigma_{Q}{ }^{2}$. In the soft-demapper the channel symbols $\underline{r}$ are demapped and ungrouped to each $M \log$-like lihood ratio (LLR) values $L_{p}^{\mu}$.

In the first iteration these a posteriori LLR values $L_{p}^{\mu}$ are deinterleaved and soft-in/soft-out decoded in a symbol-by-symbol a posteriori probability (APP) estimator; a BCJR[16] or LDPC decoder[10] depending on the used encoder. The output of the decoder delivers the APP LLR $L_{p}^{C}$. For iterative decoding it is necessary to exchange extrinsic information between the soft-demapper and the decoder only. So the soft-input LLR values of the coded bits for the soft-demapper consist of interleaved a posteriori LLR of the decoder without the a-priori information, $L_{a}^{\mu}=\prod\left\{L_{p}^{C}-L_{a}^{C}\right\}$. Respectively, the a-priori LLR for the BCJR decoder are computed as the deinterleaved extrinsic LLR of the soft-demapper, $L_{a}^{C}=\prod\left\{L_{p}^{\mu}-L_{a}^{\mu}\right\}$.

The decoder performs bitwise soft-input processing, thus the demapper extracts a soft value for each coded bit $t_{0,1, ., M-1}$ of a $2^{M}$-ary complex channel symbol $\underline{r}$. When a-priori information is available, the soft-demapper calculates the APP LLR $L_{p}^{\mu}$ of bit $k$ computes to[9]

$$
\begin{gathered}
L_{p}^{\mu}\left(t_{k} \mid \underline{r}\right)=\ln \frac{p\left(t_{k}=1 \mid \underline{r}\right)}{p\left(t_{k}=0 \mid \underline{r}\right)}=L_{a}^{\mu}\left(t_{k}\right)+ \\
\sum_{i=0}^{2^{M-1}-1} p\left(\underline{r} \mid t_{k}=1, t_{j \neq k} \equiv \operatorname{bin}(i)\right) \cdot \exp \sum_{\substack{j=0, j \neq k \\
\text { btst }(i, h)=1}}^{M-1} L_{a}^{\mu}\left(t_{j}\right) \\
\sum_{i=0}^{2^{M-1}-1} p\left(\underline{r} \mid t_{k}=0, t_{j \neq k} \equiv \operatorname{bin}(i)\right) \cdot \exp \sum_{\substack{j=0, j \neq k \\
\text { btst }(i, h)=1}}^{M-1} L_{a}^{\mu}\left(t_{j}\right)
\end{gathered}
$$

where $t_{j \neq k} \equiv \mathrm{b}$ in $(i)$ denotes the joint event of the variables $t_{j \neq k}$; $k, j \in\{0 . . M-1\}$ having the values 0,1 according to the binary decomposition of $i$. The function $\operatorname{btst}(i, h)$ takes the value 1 if bit number $h$ is set in the binary decomposition of $i$, otherwise it is 0 , where

$$
h= \begin{cases}j, & j \leq k \\ j-1, & j \geq k .\end{cases}
$$

Using the max-log approximation[8] and assuming that the received symbols are only corrupted by Gaussian, noise we can be rewritten by the soft-demapping algorithm (6) with (5) according to[8]

$$
L_{p}^{\mu}\left(t_{k} \mid \underline{r}\right) \approx L_{a}^{\mu}\left(t_{k}\right)+
$$

$$
\frac{\max _{i=0.2^{M-1}-1}\left[-\frac{1}{2 \sigma^{2}}\left|\underline{r}-\underline{a}_{k,(1, i)}\right|^{2}+\sum_{\substack{j=0, j \neq k \\ \operatorname{bstt}(i, h)=1}}^{M-1} L_{a}^{\mu}\left(t_{j}\right)\right]}{\max _{i=0.2^{M-1}-1}\left[-\frac{1}{2 \sigma^{2}}\left|\underline{r}-\underline{a}_{k,(0, i)}\right|^{2}+\sum_{\substack{j=0, j \neq k \\ \mathrm{bst}(i, h)=1}}^{M-1} L_{a}^{\mu}\left(t_{j}\right)\right]},
$$

where $\underline{a}_{k,(1, i)}=\operatorname{map}\left(t_{k}=1, t_{j \neq k} \equiv \mathrm{b}\right.$ in $\left.(i)\right)$ and $\underline{a}_{k,(0, i)}=\operatorname{map}\left(t_{k}=0\right.$, $\left.t_{j \neq k} \equiv \operatorname{bin}(i)\right), j \in\{0 . . M-1\}$. This algorithm can even be more simplified, when only the metrics to those symbols closest to the received symbol are computed, which are also the most likely ones. In the following we use the soft-demapping algorithm of (7), since we expect the soft-demapper implementation in hardware without the max-log approximation unfeasible for high data rates and the losses are inferior anyway.

\section{Design of BICM-ID for IPM}

In this section we discuss the design of BICM-ID, using a 64-IPM signal constellation and RSC codes. We restricted the use of LDPC codes and utilized RSC codes, since they offer a greater degree of freedom in design for coded modulation, when the code rate is high. As denoted above, residual errors are removed by an outer RS (2720, 2550) code. In order to design the BICM-ID scheme towards proper convergence and to acquire a post-FEC BER below the threshold BER of the outer RS code of $1.1 \cdot 10^{-3}$, we use the EXIT chart analysis, which is a powerful tool to visualize the flow of extrinsic information between the soft-demapper and decoder[9]. In the EXIT chart analysis, the mutual information (MI) of the decoder/demapper is plotted versus its a-priori input, i.e. the MI of the demapper/decoder. If an optimum demapper/decoder is used, the knowledge of the MI contained in the a-priori information is sufficient to derive the MI of the decoder/demapper. Denoting the encoder/demapper input by $X_{a}$ and the corresponding extrinsic decoder/demapper output by $X_{e}$, the MI $I\left(X_{e} ; X_{a}\right)$ calcu lates to

$$
I\left(X_{e} ; X_{a}\right)=\int_{X_{e} X_{a}} f\left(x_{e}, x_{a}\right) \log _{2} \frac{f\left(x_{e}, x_{a}\right)}{f\left(x_{e}\right) f\left(x_{a}\right)} \mathrm{d} x_{a} \mathrm{~d} x_{e}
$$

As denoted above the 256-IPM constellation offers a spectral efficiency very close to the Shannon Limit, however designing a mapping for a proper convergence of BICM-ID using either RSC or LDPC component codes is still an open problem. Therefore we restricted us to the design of BICM-ID using the 64-IPM signal constellation only. Figure 6 depicts the EXIT chart of the designed 64-IPM coded modulation scheme at a per-channel input power of $-7 \mathrm{dBm}$ after $960 \mathrm{~km}$ SSMF transmission.

The mapping was designed to be most Gray like, in order to provide a flat soft-demapper EXIT function, which is equivalent to allow a fast convergence of the iteration loop. 
The RSC code applied has a constraint length of 7 and was punctured to result in a code rate of $R_{R S C}=0.86$. The CW length was set to $n=20736$, to comply with the number of bits in two OFDM symbols. As indicated by the dashed line, the EXIT functions of soft-demapper and decoder intersect at an MI corresponding to BER $<1.1 \cdot 10^{-3}$. The obtained BERs of the designed BICM-ID system for different per-channel input powers and different number of iterations after $960 \mathrm{~km} \mathrm{SSMF}$ transmission are depicted in Figure 7.

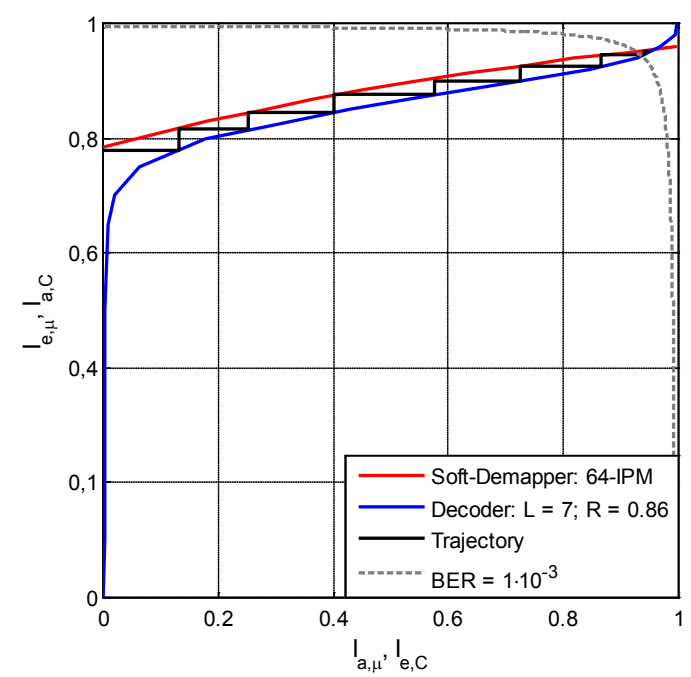

Figure 6. EXIT chart for the designed 64-IPM signal constellation and RSC code at a fiber per-channel launch power of $-7 \mathrm{dBm}$ after $960 \mathrm{~km}$ SSMF transmission



Figure 7. BER vs. per-channel input power at the decoder output of the designed coded 64-IPM scheme after $960 \mathrm{~km}$ SSMF transmission

Obviously, 6 iterations are necessary to provide a BER $<1.1 \cdot 10^{-3}$, which is sufficient for the outer EFEC coding scheme to deliver a post-FEC BER $<10^{-15}$. Considering a 6.69 FEC overhead for outer coding and $10 \%$ overhead for OFDM pilots duration and guard interval, this system obtains a spectral efficiency of $8.64 \mathrm{bit} / \mathrm{s} / \mathrm{Hz}$ in both states of polarization after $960 \mathrm{~km} \mathrm{SSMF} \mathrm{transmission.}$

\section{Coded Modulation with LDPC Codes}

For optical transponders processing at data rates beyond $400 \mathrm{Gbit} / \mathrm{s}$, another potential candidate for strong soft-decision-based FEC in BICM-ID are LDPC codes, which are linear codes defined by the sparse parity-check matrix invented by Gallager[10]. They allow for an iterative decoding procedure, denoted as belief propagation or sum-product algorithm, where extrinsic information is exchanged between variable nodes, corresponding to information and redundancy bits, and check nodes, representing the parity check equations. The edges between them are given by the parity-check matrix. An LDPC codes is specified by two degree distributions $\lambda(x)$ and $\rho(x)$, where

$\lambda(x)=\sum_{i=1}^{d_{l}} \lambda_{i} x^{i-1}$ and $\rho(x)=\sum_{j=1}^{d c} \rho_{j} x^{j-1} . \lambda_{i}$ is the fraction of edges that belong to degree- $i$ variable nodes, $\rho_{j}$ is the fraction of edges that belong to degree- $j$ check nodes, $d_{l}$ is the maximum variable node degree, and $d_{c}$ is the maximum check node degree. LDPC codes are expected to exhibit superior error-correcting performance close to the Shannon limit, which implies a flat LDPC decoder EXIT function in the EXIT chart analysis.

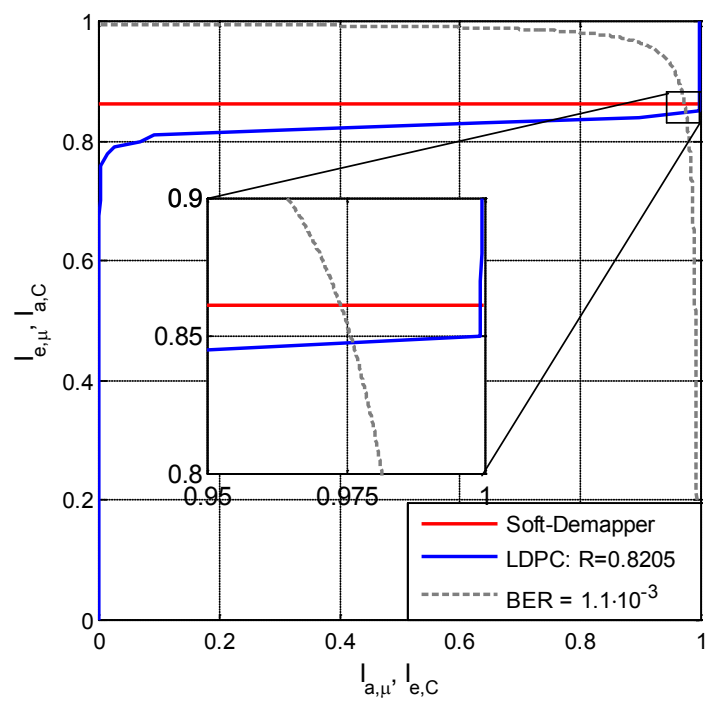

Figure 8. EXIT chart for the designed 64-QAM signal constellation and LDPC code at a fiber per-channel launch power of $-7 \mathrm{dBm}$ after $960 \mathrm{~km}$ SSMF transmission

Therefore we intend to use LDPC codes in BICM-ID when square signal constellations with Gray mapping are applied only. This offers the advantage that there is no need for information feedback in BICM-ID, since the soft-demapper EXIT-function of Gray mapped signal constellations is flat too, so there is no gain in computing the extrinsic information when a-priori information is available. Furthermore the interleavers are dispensable, since in an LDPC CW adjacent bits participate in different checks, so there are no significant statistical dependencies between the bits mapped to symbol. The degree distribution of a proper LDPC code for the system regarding the capacity of the modulation was found by means of density evolution[17]. Figure 8 shows the EXIT chart of the designed coding scheme at a per-channel input power of 
$-7 \mathrm{dBm}$ after $960 \mathrm{~km}$ SSMF transmission.

Obviously, the EXIT functions of the soft-demapper and decoder intersect at an MI corresponding to a BER $<1.1 \cdot 10^{-3}$ without performing any iterations between the decoder and demapper. The LDPC code applied has a CW length of $n=20736$, same as the RSC code before. The code rate was $R_{L D P C}=0.8205$ and 30 iterations were performed in the LDPC decoder. The check nodes of the applied LDPC code have degree 30 only, $\rho(30)=1$; the degree distribution of the variable nodes are denoted in Table 2.

Table 2. LDPC code parameters; $R_{L D P C}=0.8205 ; 30$ iterations; $\rho(30)=1$

\begin{tabular}{|c|c|c|c|c|}
\hline $\mathrm{x}$ & $\lambda(\mathrm{x})$ & & $\mathrm{x}$ & $\lambda(\mathrm{x})$ \\
\hline 2 & 0.124799 & & 25 & 0.008666 \\
\hline 3 & 0.276860 & & 28 & 0.033088 \\
\hline 7 & 0.076933 & & 37 & 0.001093 \\
\hline 8 & 0.042432 & & 69 & 0.051768 \\
\hline 9 & 0.007960 & & 74 & 0.122933 \\
\hline 19 & 0.011330 & & 77 & 0.028724 \\
\hline 20 & 0.134332 & & 80 & 0.040174 \\
\hline 23 & 0.038905 & & & \\
\hline
\end{tabular}

The available BERs after performing one and two iterations of the iterative soft-demapping and decoding system versus different per-channel input powers after $960 \mathrm{~km} \mathrm{SSMF}$ transmission are depicted in Figure 9.

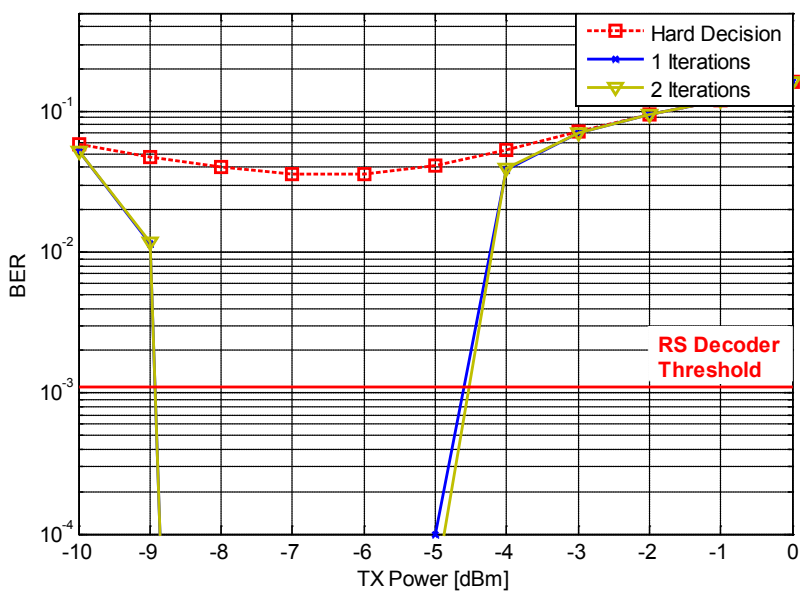

Figure 9. BER vs. per-channel input power at the decoder output of the designed coded 64-QAM scheme after $960 \mathrm{~km}$ SSMF transmission

As expected by the EXIT chart analysis, there is no significant gain when performing iterations between LDPC decoder and soft-demapper. Furthermore, the error floor of the inner coding system is several decades blow the threshold BER of the RS $(2720,2500)$ code, so it is reasonable to consider the simpler RS $(255,239)$ code with an NCG of $6.2 \mathrm{~dB}$ for an post-FEC BER of $5 \cdot 10^{-15}$ for outer coding.

Considering the $6.69 \%$ overhead for outer coding and $10 \%$ overhead for OFDM pilots duration and guard interval, a spectral efficiency of $8.24 \mathrm{bit} / \mathrm{s} / \mathrm{Hz}$ was achieved with the system in both states of polarization after $960 \mathrm{~km}$ SSMF transmission.

\section{Conclusions}

In this paper we presented a simple, but spectral highly efficient coding scheme for optical OFDM-based communications, relying on the concatenation of BICM-ID with a high-rate outer code to obtain a post-FEC BER < $10^{-15}$. Further, we provided design principles of capacity approaching constellations and showed how to jointly optimize mapping and coding for a proper convergence of the iteration loop.

We demonstrate that non-uniform constellations based on IPQ outperform conventional square signal constellations. In order to compare the performance of IPM versus QAM towards BER performance we developed different coding schemes, showing that in both cases an overall post-FEC BER $<10^{-15}$ is achievable, but, when using coded 64-IPM a spectral efficiency of $8.64 \mathrm{bit} / \mathrm{s} / \mathrm{Hz}$ in both states of polarization after $960 \mathrm{~km}$ SSMF transmission could be obtained, which is $\approx 0.4 \mathrm{bit} / \mathrm{s} / \mathrm{Hz}$ greater compared to coded 64-QAM.

However for a practical implementation we suggest coded modulation schemes, based on QAM constellations and LDPC codes, to be more appropriate, since they are implementable more efficiently in hardware to operate at high data rates. Dis regarding the loss in spectral efficiency due to OFDM pilots duration and guard interval, th is coding scheme is able to approach the Shannon limit by 1.85 $\mathrm{bit} / \mathrm{s} / \mathrm{Hz}$ in both states of polarization.

\section{ACKNOWLEDGEMENTS}

This work was supported by the German Research Foundation (DFG) under grant UR 75/3-1.

\section{REFERENCES}

[1] R. Nagarajan, D. Lambert, M. Kato, V. Lal, G. Goldfarb, J. Rahn, et. al., "10 Channel, 100Gbit/s per Channel, Dual Polarization, Coherent QPSK, Monolithic InP Receiver Photonic Integrated Circuit", in Proc. OFC/NFOEC, OML7, San Diego, CA, USA, 2011.

[2] T. Lotz, W. Sauer-Greff and R. Urbansky, "Capacity Approaching Coded Modulation in Optical Communications," Proc. 13 ${ }^{\text {th }}$ International Conference on Transparent Optical Networks (ICTON), Stockholm, Sweden 2011.

[3] Z. H. Peric, I. B. Djordjevic, S. M. Bogosavljevic and M. C. Stefanovic, "Design of Signal Constellations for Gaussian Channel by Iterative Polar Quantization," in Proc. 9th Mediterranean Electrotechnical Conference 2, pp. 866-869, 18-20 May 1998; Tel-Aviv, Israel.

[4] C. Berrou, A. Glavieux, and P. Thitimajshima, "Near Shannon limit error correcting coding and decoding: Turbo-Codes," Proc. IEEE ICC, pp. 1064-1070, 1993.

[5] I. B. Djordjevic, H. G. Batshon, L. Xu and T. Wang, "Coded 
Polarization-Multiplexed Iterative Polar Modulation (PM-IPM) for Beyond $400 \mathrm{~Gb} / \mathrm{s}$ Serial Optical Transmission," in Proc. OFC/NFOEC, OMK2, San Diego, CA, USA, 2010.

[6] I. B. Djordjevic, M. Arabaci, and L. Minkov, "Next Generation FEC for High-Capacity Communication in Optical Transport Networks," J. Lightw. Technol. 27, 3518-3530 (2009).

[7] X. Liu, S. Chandrasekhar, Z. Benyuan, P. J. Winzer, A. H. Gnauck, D.W. Peckham, "448-Gb/s Reduced-Guard-Interval CO-OFDM Transmission Over 2000 km of Ultra-Large-Area Fiber and Five 80-GHz-Grid ROADMs," Journal of Lightwave Technology, 2011

[8] S. ten Brink, J. Speidel and R.-H. Yan, "Iterative Demapping and Decoding for Multilevel Modulation," Proc. IEEE GLOBECOM, 1998.

[9] S. ten Brink, "Convergence Behavior of Iteratively Decoded Parallel Concatenated Codes," IEEE Trans. Commun., vol. 49, 2001.

[10] R. G. Gallager, Information Theory and Reliable Communication, New York: Wiley, 1968.

[11] D. Raphaeli and A. Gurevitz, "Constellation Shaping for Pragmatic Turbo-Coded Modulation with High Spectral Efficiency," IEEE Trans. Commun., vol. 52,p. 341345, 2004.
[12] H. G. Batshon, I. B. Djordjevic, L. Xu and T. Wang, "Iterative Polar Quantization-Based Modulation to Achieve Channel Capacity in Ultrahigh-Speed Optical Communication Systems," IEEE Photonics Journal, vol. 2, 2010.

[13] M. Mayrock and H. Haunstein, "Spectral Efficiency Limitation by Fiber Non-linearity in Optical OFDM Transmission Systems, " in Proceedings of 14th International OFDM Workshop, Hamburg, Germany, September 2009.

[14] M. Mayrock, "Analysis and Compensation of Non-linear Signal Distortion in Optical OFDM," 15th International OFDM-Workshop, pp. 56-60, 2010.

[15] International Telecommunication Union, "Interfaces for the Optical Transport Network," Telecommunication Standardization Sector, G.709, 2003.

[16] International Telecommunication Union, "Forward error correction for high bit-rate DWDM submarine systems," Telecommunication Standardization Sector, G.975.1, 2004.

[17] L. Bahl, J. Cocke, F Jelinek and J. Raviv, "Optimal Decoding of Linear Codes for Minimizing Symbol Error Rate, " IEEE Trans. Inf. Theory, vol. 20, 1974.

[18] S.-Y. Chung, R.J. Richardson, and R.L. Urbanke, "Analy sis of sum-product decoding of low-density parity-check codes using a Gaussian approximation," IEEE Trans. Inf. Theory, vol. 47, 2001. 